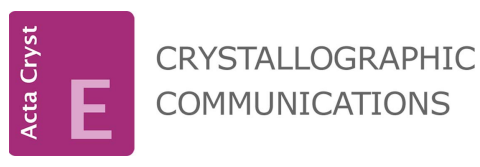

ISSN 2056-9890

Received 2 February 2015

Accepted 3 March 2015

Edited by J. Simpson, University of Otago, New Zealand

₹ Additional correspondence author, email: suchada.c@psu.ac.th. Thomson Reuters ResearcherID: A-5085-2009.

Keywords: crystal structure; Pd" complex; NO donors; Schiff base; catalyst activity; hydrogen bonding; $\pi-\pi$ interactions

CCDC reference: 1045879

Supporting information: this article has supporting information at journals.iucr.org/e

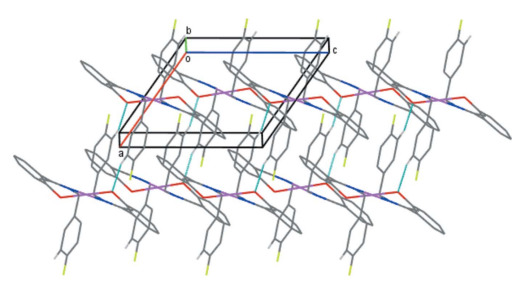

\section{Crystal structure of bis(2-\{1-[(E)-(4-fluorobenzyl)- imino]ethyl\}phenolato- $\kappa^{2} N, O$ ) palladium(II)}

\author{
Amalina Mohd Tajuddin, ${ }^{a}$ Hadariah Bahron, ${ }^{\mathrm{a}, \mathrm{b} *}$ Hamizah Mohd Zaki, ${ }^{\mathrm{a}}$ Karimah \\ Kassim $^{a, c}$ and Suchada Chantrapromma ${ }^{d} \ddagger$
}

${ }^{\mathbf{a}}$ Faculty of Applied Sciences, Universiti Teknologi MARA, 40450 Shah Alam, Selangor, Malaysia, ${ }^{\mathbf{b}}$ DDH CoRe, Universiti Teknologi MARA, 40450 Shah Alam, Selangor, Malaysia, ' Institute of Science, Universiti Teknologi MARA, 40450 Shah Alam, Selangor, Malaysia, and dDepartment of Chemistry, Faculty of Science, Prince of Songkla University, Hat-Yai, Songkhla 90112, Thailand. *Correspondence e-mail: hadariah@salam.uitm.edu.my

The asymmetric unit of the title complex, $\left[\mathrm{Pd}\left(\mathrm{C}_{15} \mathrm{H}_{13} \mathrm{FNO}\right)_{2}\right]$, contains one half of the molecule with the $\mathrm{Pd}^{\mathrm{II}}$ cation lying on an inversion centre and is coordinated by the bidentate Schiff base anion. The geometry around the cationic $\mathrm{Pd}^{\mathrm{II}}$ centre is distorted square planar, chelated by the imine $\mathrm{N}$ - and phenolate O-donor atoms of the two Schiff base ligands. The $\mathrm{N}$ - and O-donor atoms of the two ligands are mutually trans, with $\mathrm{Pd}-\mathrm{N}$ and $\mathrm{Pd}-\mathrm{O}$ bond lengths of 2.028 (2) and 1.9770 (18) $\AA$, respectively. The fluorophenyl ring is tilted away from the coordination plane and makes a dihedral angle of $66.2(2)^{\circ}$ with the phenolate ring. In the crystal, molecules are linked into chains along the [101] direction by weak $\mathrm{C}-\mathrm{H} \cdots \mathrm{O}$ hydrogen bonds. Weak $\pi-\pi$ interactions with

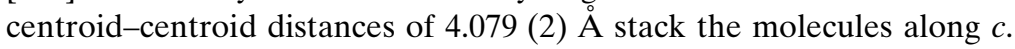

\section{Chemical context}

Schiff bases represent one of the most widely utilized classes of ligands in coordination chemistry and the chemistry of Schiff bases is still an area of increasing interest (Canali \& Sherrigton, 1999). The $\mathrm{Pd}^{\mathrm{II}}$ and $\mathrm{Ni}^{\mathrm{II}}$ complexes of Schiff bases have attracted much attention as they play important roles in bioinorganic chemistry and may provide the basis for models of active sites of biological systems (Malik et al., 2013) or act as catalysts (Shahnaz et al., 2013). The title compound, $\left[\mathrm{Pd}\left(\mathrm{C}_{15} \mathrm{H}_{13} \mathrm{FNO}\right)_{2}\right]$, is related to the previously reported compound bis\{2-[(E)-(4-fluorobenzyl)iminomethyl]phenolato- $\kappa^{2} N, O^{1}$ \}nickel(II) (Mohd Tajuddin et al., 2014) in terms of the coordination geometry around the central metal. In this article, we report the synthesis of the title Schiff base- $\mathrm{Pd}^{\mathrm{II}}$ complex and its characterization by spectroscopy and elemental analysis. The X-ray structure (Fig. 1), confirms the binding mode of the 4-fluorobenzyl(iminoethyl)phenolate ligand to the $\mathrm{Pd}^{\mathrm{II}}$ cation.

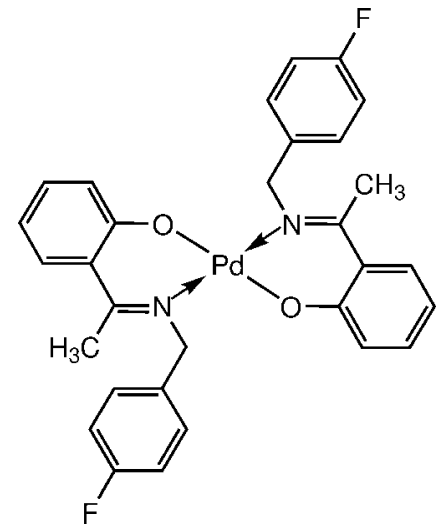




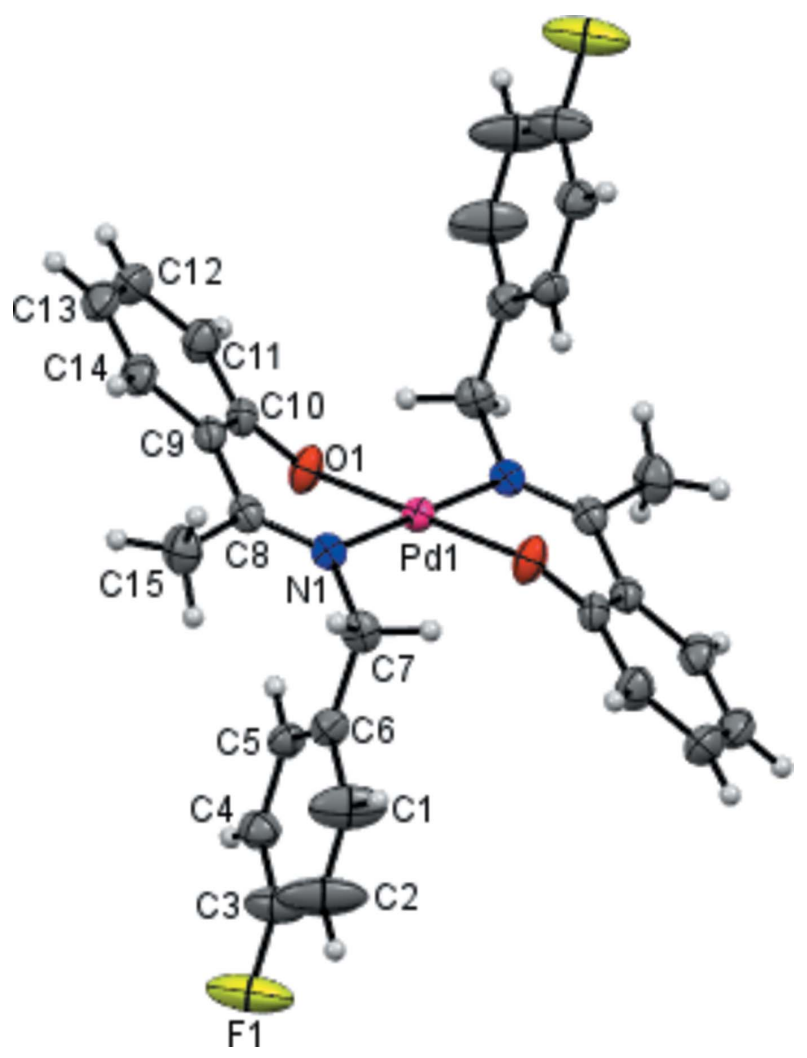

Figure 1

The molecular structure of (1), showing $50 \%$ probability displacement ellipsoids and the atom-numbering scheme. The labelled atoms are related to the unlabelled atoms of the Schiff base ligands by the symmetry code $1-x, 2-y, 2-z$

The title compound (1) was screened for catalytic activity in the Suzuki cross-coupling reaction between phenylboronic acid and iodobenzene with a catalyst loading of $1 \mathrm{mmol} \%$. The conversion of iodobenzene was found to occur with a yield of $52 \%$.

\section{Structural commentary}

The asymmetric unit of (1) contains one-half of the molecule with the $\mathrm{Pd}^{\mathrm{II}}$ cation lying on an inversion centre and the Schiff base anion acting as an $N, O$ bidentate chelate ligand (Fig. 1).

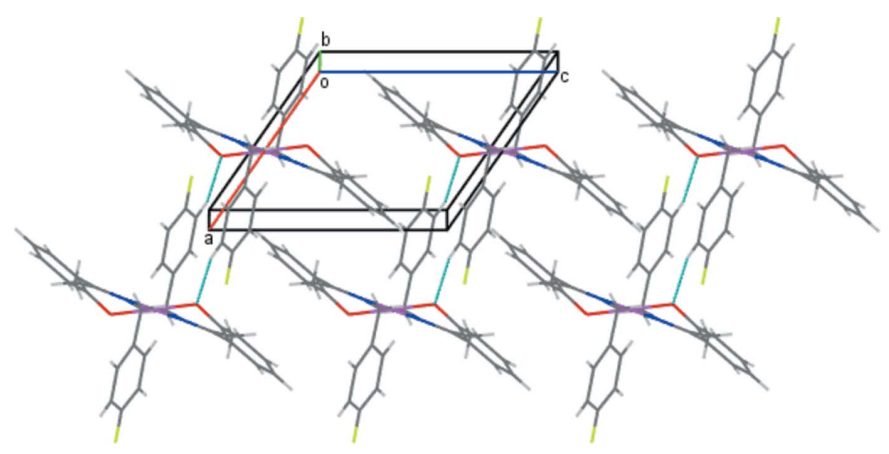

Figure 2

Screw chains of molecules of (1) linked by $\mathrm{C}-\mathrm{H} \cdots \mathrm{O}$ interactions (drawn as dashed lines).
Table 1

Selected geometric parameters $\left(\AA{ }^{\circ}{ }^{\circ}\right)$.

\begin{tabular}{lclc}
\hline Pd1-O1 & $1.9770(18)$ & Pd1-N1 & $2.028(2)$ \\
O1-Pd1-N1 & $88.48(8)$ & O1 1 - Pd1-N1 & $91.52(8)$ \\
\hline
\end{tabular}

Symmetry code: (i) $-x+1,-y+2,-z+2$.

Table 2

Hydrogen-bond geometry $\left(\AA,^{\circ}\right)$.

\begin{tabular}{lllll}
\hline$D-\mathrm{H} \cdots A$ & $D-\mathrm{H}$ & $\mathrm{H} \cdots A$ & $D \cdots A$ & $D-\mathrm{H} \cdots A$ \\
\hline $\mathrm{C} 4-\mathrm{H} 4 A \cdots \mathrm{O} 1^{\mathrm{ii}}$ & 0.93 & 2.50 & $3.405(5)$ & 165
\end{tabular}

Symmetry code: (ii) $-x+2,-y+2,-z+2$.

The $\mathrm{Pd}^{\mathrm{II}}$ cation binds to the $\mathrm{N}$ and the $\mathrm{O}$ atoms of two symmetry-related Schiff base ligand such that the $\mathrm{N}$ and $\mathrm{O}$ atoms are mutually trans. The $\mathrm{N}_{2} \mathrm{O}_{2}$ donor sets of the two chelating Schiff base ligands in the equatorial plane around Pd1 adopt a slightly distorted square-planar coordination geometry. The Pd1-N1 and Pd1-O1 distances (Table 1) are typical of square-planar $\mathrm{Pd}^{\mathrm{II}}$ complexes, and compare well with those observed in other closely related $\mathrm{Pd}^{\mathrm{II}}$ complexes (Adrian et al., 2008; Bahron et al., 2014; Wan Ibrahim \& Shamsuddin, 2012). The bite angle of the iminomethylphenolate chelate, $\mathrm{N} 1-\mathrm{Pd} 1-\mathrm{O} 1$ is $88.48(8)^{\circ}$, which is also similar to that in a related $\mathrm{Pd}^{\mathrm{II}}$ complex (Bahron et al., 2014). The ring $\mathrm{Pd} 1 / \mathrm{N} 1 / \mathrm{C} 8 / \mathrm{C} 9 / \mathrm{C} 10 / \mathrm{O} 1$ adopts an envelope conformation with Pd1 displaced by 0.270 (2) $\AA$ from the plane of the other ring atoms, and with puckering parameters $Q=$ 0.525 (2) $\AA, \theta=112.8$ (3) and $\varphi=206.9$ (3) $)^{\circ}$. Other bond lengths and angles observed in the structure are also normal. The fluorophenyl ring (C1-C6) makes a dihedral angle of $66.2(2)^{\circ}$ with the phenolate ring (C9-C14).

\section{Supramolecular features}

In the crystal packing of (1), the molecules are linked into chains along the [101] direction by weak $\mathrm{C} 4-\mathrm{H} 4 A \cdots \mathrm{O} 1$ interactions (Fig. 2, Table 2). A weak $\pi-\pi$ stacking interaction occurs between the phenolate rings of adjacent complexes (Fig. 3), with a centroid-centroid distance, $C g 4 \cdots C g 4^{\text {iii }}$, of 4.079 (2) $\AA$ [symmetry code: (iii) $=1-x, 2-y, 1-z$; $C g 4$ is the centroid of the $\mathrm{C} 9-\mathrm{C} 14$ ring]. These combine with the $\mathrm{C}-$ $\mathrm{H}$... O contacts to generate sheets in the $a c$ plane (Fig. 4). These sheets are further stacked along the $b$-axis direction.

\section{Database survey}

Six $\mathrm{Pd}^{\mathrm{II}}$ complexes with related Schiff base $\mathrm{N}_{2} \mathrm{O}_{2}$ donor sets have been reported (Brunner et al., 2000; Mehta \& Vengurlekar, 2001; Bahron et al., 2011, 2014; Mohd Tajuddin et al., 2012a; Tsuno et al., 2013). However, only three of these $\mathrm{Pd}^{\mathrm{II}}$ complexes have closely related Schiff base ligands (Bahron et al., 2011; 2014; Mohd Tajuddin et al., 2012a). 


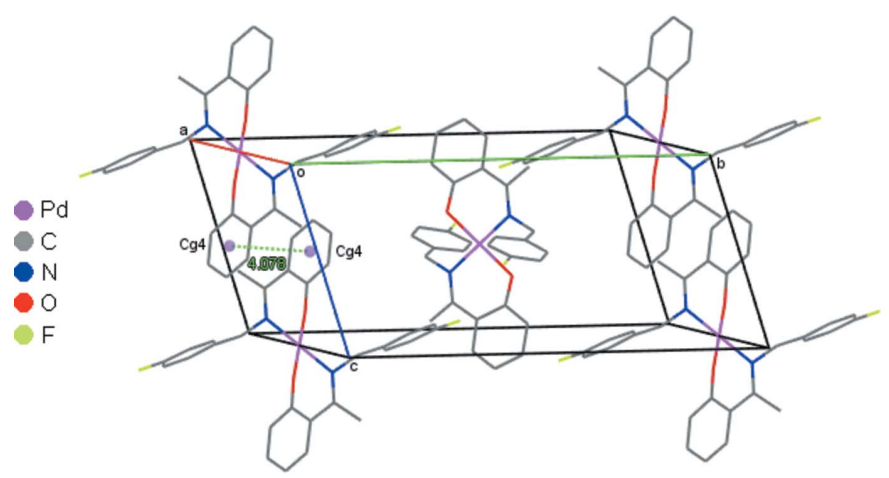

Figure 3

$\pi-\pi$ contacts for (1) drawn as dotted lines with ring centroids shown as coloured spheres. $\mathrm{Cg} 4$ is the centroid of the $\mathrm{C} 9-\mathrm{C} 14$ ring. $\mathrm{H}$ atoms are omitted for clarity.

\section{Synthesis and crystallization}

The ligand, (E)-2-(1-(4-fluorobenzylimino)ethyl)phenol (Mohd Tajuddin et al., 2012b) ( $2 \mathrm{mmol}, 0.4877 \mathrm{~g}$ ) was dissolved in $\mathrm{CH}_{3} \mathrm{CN}(30 \mathrm{~mL})$ in a round-bottomed flask. Palladium(II) acetate (1 mmol, $0.2251 \mathrm{~g})$ was dissolved separately in $\mathrm{CH}_{3} \mathrm{CN}(20 \mathrm{~mL})$ and was then added into the flask containing the ligand solution. The mixture was stirred and refluxed for $5 \mathrm{~h}$ upon which a turmeric yellow solid was formed. The solid was filtered off, washed with ice-cold $\mathrm{CH}_{3} \mathrm{CN}$ and air dried at room temperature. The solid product was recrystallized from chloroform, yielding yellow crystals (yield 48.5\%). ${ }^{1} \mathrm{H}$ NMR,${ }^{13} \mathrm{C}$ NMR and IR spectral bands have been studied and agree well with the structure obtained from the values of the $\mathrm{CHN}$ analyses and X-ray structure determination.

Melting point $508-510 \mathrm{~K}$. Analytical data for $\mathrm{C}_{30} \mathrm{H}_{26} \mathrm{~F}_{2} \mathrm{~N}_{2} \mathrm{O}_{2} \mathrm{Pd}$ : C, 60.97; H, 4.43; N, 4.74\%; Found: C, 60.81; $\mathrm{H}, 4.49 ; \mathrm{N}, 4.66 \%$. IR $\left(\mathrm{KBr}, \mathrm{cm}^{-1}\right): 1598 v(\mathrm{C}=\mathrm{N}), 1319$ $v(\mathrm{C}-\mathrm{N}), 1216 v(\mathrm{C}-\mathrm{O}), 1321 v\left(\mathrm{CH}_{3}\right), 556 v(\mathrm{Pd}-\mathrm{N}), 450$ $v(\mathrm{Pd}-\mathrm{O}) .{ }^{1} \mathrm{H}$ NMR $\left(300 \mathrm{MHz}, \mathrm{CDCl}_{3}\right): \delta$ (p.p.m.) $2.32(\mathrm{~s}, 3 \mathrm{H}$, $\left.\mathrm{C}-\mathrm{CH}_{3}\right), 5.11\left(s, 2 \mathrm{H}, \mathrm{CH}_{2}\right), 6.53-7.46$ (ArC). ${ }^{13} \mathrm{C} \mathrm{NMR}$ (300 $\left.\mathrm{MHz}, \mathrm{CDCl}_{3}\right)$ : $\delta$ (p.p.m.) $19.5\left(\mathrm{C}-\mathrm{CH}_{3}\right), 54.2\left(\mathrm{CH}_{2}\right)$, 115.3, 115.6, 121.3, 128.6, $130.2($ ArC $), 169.8(\mathrm{C}=\mathrm{N})$.

The infrared spectrum of (1) exhibits a strong band at $1598 \mathrm{~cm}^{-1}$ assignable to the $\mathrm{C}=\mathrm{N}$ stretching frequency of the azomethine moiety. Weak bands at 556 and $450 \mathrm{~cm}^{-1}$ attributable to $\mathrm{Pd}-\mathrm{N}$ and $\mathrm{Pd}-\mathrm{O}$ vibrations, respectively (Ouf et al., 2010), are due to the participation of the nitrogen of the azomethine group and the oxygen of the phenolate ring in the complexation of the palladium(II) centre by the Schiff base ligands. From the NMR results, the free 4-fluorobenzyl(iminoethyl)phenolate ligand shows a multiplet at around 6.80-7.57 p.p.m. assignable to the aromatic protons. A corresponding multiplet appears in almost the same position in the spectrum of the $\mathrm{Pd}^{\mathrm{II}}$ complex (compound 1) as that observed by Gupta et al. (2013). Singlets for aliphatic methylene $\left(-\mathrm{CH}_{2}\right)$ and methyl $\left(-\mathrm{CH}_{3}\right)$ protons appear at 5.11 and 2.32 p.p.m., respectively. The ${ }^{13} \mathrm{C}$ chemical shift for the imine carbon $(\mathrm{C}=\mathrm{N})$ is found at 169.8 p.p.m., agreeing with data reported by Şenol et al. (2011).
Table 3

Experimental details.

\begin{tabular}{|c|c|}
\hline \multicolumn{2}{|l|}{ Crystal data } \\
\hline Chemical formula & {$\left[\mathrm{Pd}\left(\mathrm{C}_{15} \mathrm{H}_{13} \mathrm{FNO}\right)_{2}\right]$} \\
\hline$M_{\mathrm{r}}$ & 590.93 \\
\hline Crystal system, space group & Monoclinic, $P 2_{1} / c$ \\
\hline Temperature $(\mathrm{K})$ & 296 \\
\hline$a, b, c(\AA)$ & $7.5924(5), 21.9212(14), 9.3475(5)$ \\
\hline$\beta\left(^{\circ}\right)$ & $124.963(4)$ \\
\hline$V\left(\AA^{3}\right)$ & $1274.97(15)$ \\
\hline$Z$ & 2 \\
\hline Radiation type & Мо $K \alpha$ \\
\hline$\mu\left(\mathrm{mm}^{-1}\right)$ & 0.77 \\
\hline Crystal size $(\mathrm{mm})$ & $0.50 \times 0.25 \times 0.25$ \\
\hline \multicolumn{2}{|l|}{ Data collection } \\
\hline Diffractometer & $\begin{array}{l}\text { Bruker APEXII CCD area } \\
\text { detector }\end{array}$ \\
\hline Absorption correction & $\begin{array}{l}\text { Multi-scan (SADABS; Bruker, } \\
\text { 2009) }\end{array}$ \\
\hline$T_{\min }, T_{\max }$ & $0.699,0.830$ \\
\hline $\begin{array}{l}\text { No. of measured, independent and } \\
\text { observed }[I>2 \sigma(I)] \text { reflections }\end{array}$ & $38866,2776,2720$ \\
\hline$R_{\text {int }}$ & 0.057 \\
\hline$(\sin \theta / \lambda)_{\max }\left(\AA^{-1}\right)$ & 0.639 \\
\hline \multicolumn{2}{|l|}{ Refinement } \\
\hline$R\left[F^{2}>2 \sigma\left(F^{2}\right)\right], w R\left(F^{2}\right), S$ & $0.034,0.073,1.30$ \\
\hline No. of reflections & 2776 \\
\hline No. of parameters & 170 \\
\hline $\mathrm{H}$-atom treatment & H-atom parameters constrained \\
\hline$\Delta \rho_{\max }, \Delta \rho_{\min }\left(\mathrm{e} \AA^{-3}\right)$ & $0.24,-0.48$ \\
\hline
\end{tabular}

Computer programs: APEX2 and SAINT (Bruker, 2009), SHELXTL (Sheldrick, 2008), PLATON (Spek, 2009), Mercury (Macrae et al., 2006) and publCIF (Westrip, 2010).

The title compound was screened for catalytic activity in the Suzuki cross-coupling reaction between phenylboronic acid with iodobenzene. The reaction was carried out under nitrogen at $373 \mathrm{~K}$ in dimethylacetamide with a catalyst loading of $1 \mathrm{mmol} \%$. The conversion of iodobenzene was monitored using GC-FID after 24 hours of reaction time. This resulted in a $52 \%$ conversion of iodobenzene in the reaction.

\section{Refinement}

Crystal data, data collection and crystal structure refinement details are summarized in Table 3. All $\mathrm{H}$ atoms were posi-

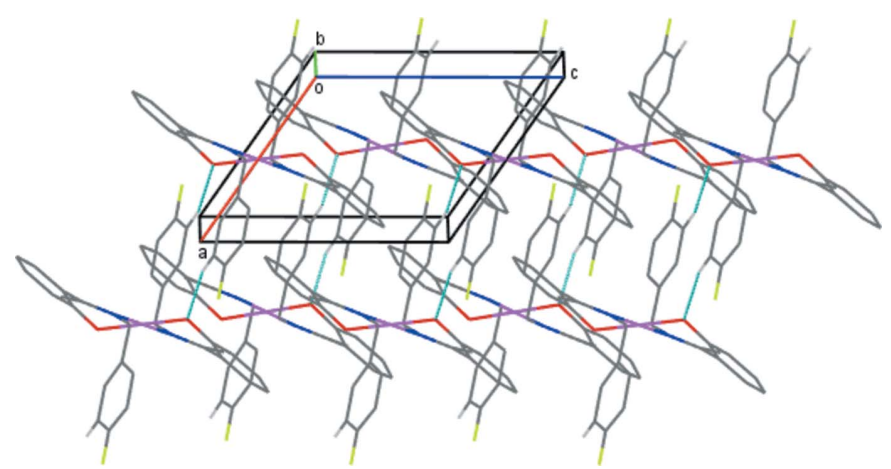

Figure 4

The packing of (1) viewed approximately along the $b$ axis showing molecular sheets of the $\mathrm{Pd}^{\mathrm{II}}$ complex. Only $\mathrm{H}$ atoms involved in $\mathrm{C}-$ $\mathrm{H} \cdots \mathrm{O}$ interactions are shown for clarity. 
tioned geometrically and allowed to ride on their parent atoms, with $d(\mathrm{C}-\mathrm{H})=0.93 \AA$ for aromatic, $0.97 \AA$ for $\mathrm{CH}_{2}$ and $0.96 \AA$ for $\mathrm{CH}_{3}$ hydrogen atoms. The $U_{\text {iso values were }}$ constrained to be $1.5 U_{\text {eq }}$ of the carrier atom for methyl $\mathrm{H}$ atoms and $1.2 U_{\mathrm{eq}}$ for the remaining $\mathrm{H}$ atoms. A rotating group model was used for the methyl groups.

\section{Acknowledgements}

The authors wish to express their appreciation to the Universiti Teknologi MARA for the research grants Nos. 600RMI/DANA 5/3/CIFI (1/2014) and 600-RMI/DANA 5/3/PSI (1/2014) and the research facilities. A scholarship from the Universiti Teknologi MARA and the Ministry of Education Malaysia is also gratefully acknowledged.

\section{References}

Adrian, R. A., Broker, G. A., Tiekink, E. R. T. \& Walmsley, J. A. (2008). Inorg. Chim. Acta, 361, 1261-1266.

Bahron, H., Mohd Tajuddin, A., Wan Ibrahim, W. N., Hemamalini, M. \& Fun, H.-K. (2011). Acta Cryst. E67, m759-m760.

Bahron, H., Mohd Tajuddin, A., Wan Ibrahim, W. N., Chantrapromma, S. \& Fun, H.-K. (2014). Acta Cryst. E70, m289-m290.

Bruker (2009). APEX2, SAINT and SADABS. Bruker AXS Inc., Madison, Wisconsin, USA.

Brunner, H., Niemetz, M. \& Zabel, M. (2000). Z. Naturforsch. Teil B, 55, 145-154.
Canali, L. \& Sherrington, D. C. (1999). Chem. Soc. Rev. 28, 85-93. Gupta, M., Sihag, S., Varshney, A. K. \& Varshney, S. (2013). J. Chem. pp. 1-8.

Macrae, C. F., Edgington, P. R., McCabe, P., Pidcock, E., Shields, G. P., Taylor, R., Towler, M. \& van de Streek, J. (2006). J. Appl. Cryst. 39, 453-457.

Malik, S., Ghosh, S., Jain, B., Singh, A. \& Bhattacharya, M. (2013). Int. J. Inorg. Chem. pp. 1-6.

Mehta, B. H. \& Vengurlekar, V. P. (2001). Asian J. Chem. 13, 939-943. Mohd Tajuddin, A. M., Bahron, H., Kassim, K., Wan Ibrahim, W. N. \& Fun, H.-K. (2012a). Adv. Mater. Res. 554-556, 736-740.

Mohd Tajuddin, A., Bahron, H., Kassim, K., Wan Ibrahim, W. N. \& Yamin, B. M. (2012b). Malaysian J. Anal. Sci. 16, 79-87.

Mohd Tajuddin, A., Bahron, H., Mohammad Hanafiah, R., Ibrahim, N., Fun, H.-K. \& Chantrapromma, S. (2014). Acta Cryst. E70, 252 255.

Ouf, A. E., Ali, M. S., Saad, E. M. \& Mostafa, S. I. (2010). J. Mol. Struct. 973, 69-75.

Şenol, C., Hayvali, Z., Dal, H. \& Hökelek, T. (2011). J. Mol. Struct. 997, 53-59.

Shahnaz, N., Banik, B. \& Das, P. (2013). Tetrahedron Lett. 54, 28862889.

Sheldrick, G. M. (2008). Acta Cryst. A64, 112-122.

Spek, A. L. (2009). Acta Cryst. D65, 148-155.

Tsuno, T., Iwabe, H. \& Brunner, H. (2013). Inorg. Chim. Acta, 400, 262-266.

Wan Ibrahim, W. N. \& Shamsuddin, M. (2012). Cryst. Struct. Theor. Appl. 1, 25-29.

Westrip, S. P. (2010). J. Appl. Cryst. 43, 920-925. 


\section{supporting information}

Acta Cryst. (2015). E71, 350-353 [doi:10.1107/S2056989015004405]

Crystal structure of bis(2-\{1-[(E)-(4-fluorobenzyl)imino] ethyl\}phenolato$\left.\kappa^{2} N, O\right)$ palladium(II)

Amalina Mohd Tajuddin, Hadariah Bahron, Hamizah Mohd Zaki, Karimah Kassim and Suchada Chantrapromma

Computing details

Data collection: APEX2 (Bruker, 2009); cell refinement: APEX2 (Bruker, 2009); data reduction: SAINT (Bruker, 2009); program(s) used to solve structure: SHELXTL (Sheldrick, 2008); program(s) used to refine structure: SHELXTL (Sheldrick, 2008); molecular graphics: SHELXTL (Sheldrick, 2008); software used to prepare material for publication: SHELXTL (Sheldrick, 2008), PLATON (Spek, 2009), Mercury (Macrae et al., 2006) and publCIF (Westrip, 2010).

$\operatorname{Bis}\left(2-\left\{1-\left[(E)-\left(4-\right.\right.\right.\right.$ fluorobenzyl)imino] ethyl\}phenolato- $\left.\kappa^{2} N, O\right)$ palladium(II)

Crystal data

$\left[\mathrm{Pd}\left(\mathrm{C}_{15} \mathrm{H}_{13} \mathrm{FNO}\right)_{2}\right]$

$M_{r}=590.93$

Monoclinic, $P 2_{1} / c$

Hall symbol: -P $2 \mathrm{ybc}$

$a=7.5924(5) \AA$

$b=21.9212(14) \AA$

$c=9.3475(5) \AA$

$\beta=124.963(4)^{\circ}$

$V=1274.97(15) \AA^{3}$

$Z=2$

\section{Data collection}

Bruker APEXII CCD area-detector diffractometer

Radiation source: sealed tube

Graphite monochromator $\varphi$ and $\omega$ scans

Absorption correction: multi-scan

(SADABS; Bruker, 2009)

$T_{\min }=0.699, T_{\max }=0.830$

Refinement

Refinement on $F^{2}$

Least-squares matrix: full

$R\left[F^{2}>2 \sigma\left(F^{2}\right)\right]=0.034$

$w R\left(F^{2}\right)=0.073$

$S=1.30$

2776 reflections

170 parameters
$F(000)=600$

$D_{\mathrm{x}}=1.539 \mathrm{Mg} \mathrm{m}^{-3}$

Melting point $=508-510 \mathrm{~K}$

Mo $K \alpha$ radiation, $\lambda=0.71073 \AA$

Cell parameters from 2776 reflections

$\theta=3.2-27.0^{\circ}$

$\mu=0.77 \mathrm{~mm}^{-1}$

$T=296 \mathrm{~K}$

Block, yellow

$0.50 \times 0.25 \times 0.25 \mathrm{~mm}$

38866 measured reflections

2776 independent reflections

2720 reflections with $I>2 \sigma(I)$

$R_{\text {int }}=0.057$

$\theta_{\max }=27.0^{\circ}, \theta_{\min }=3.2^{\circ}$

$h=-9 \rightarrow 9$

$k=-28 \rightarrow 28$

$l=-11 \rightarrow 11$

0 restraints

Primary atom site location: structure-invariant direct methods

Secondary atom site location: difference Fourier map

Hydrogen site location: inferred from neighbouring sites 
H-atom parameters constrained

$w=1 /\left[\sigma^{2}\left(F_{\mathrm{o}}^{2}\right)+(0.0086 P)^{2}+1.3994 P\right]$

where $P=\left(F_{\mathrm{o}}^{2}+2 F_{\mathrm{c}}^{2}\right) / 3$

$$
\begin{aligned}
& (\Delta / \sigma)_{\max }<0.001 \\
& \Delta \rho_{\max }=0.24 \mathrm{e}^{-3} \\
& \Delta \rho_{\min }=-0.48 \mathrm{e} \AA^{-3}
\end{aligned}
$$

Special details

Geometry. All esds (except the esd in the dihedral angle between two 1.s. planes) are estimated using the full covariance matrix. The cell esds are taken into account individually in the estimation of esds in distances, angles and torsion angles; correlations between esds in cell parameters are only used when they are defined by crystal symmetry. An approximate (isotropic) treatment of cell esds is used for estimating esds involving 1.s. planes.

Refinement. Refinement of $\mathrm{F}^{2}$ against ALL reflections. The weighted R-factor $\mathrm{wR}$ and goodness of fit $\mathrm{S}$ are based on $\mathrm{F}^{2}$, conventional R-factors $\mathrm{R}$ are based on $\mathrm{F}$, with $\mathrm{F}$ set to zero for negative $\mathrm{F}^{2}$. The threshold expression of $\mathrm{F}^{2}>2$ sigma $\left(\mathrm{F}^{2}\right)$ is used only for calculating R-factors (gt) etc. and is not relevant to the choice of reflections for refinement. R-factors based

\begin{tabular}{|c|c|c|c|c|}
\hline & $x$ & $y$ & $z$ & $U_{\text {iso }} * / U_{\text {eq }}$ \\
\hline $\mathrm{Pd} 1$ & 0.5000 & 1.0000 & 1.0000 & $0.03202(9)$ \\
\hline F1 & $1.3139(4)$ & $0.77987(11)$ & $1.2186(5)$ & $0.1153(11)$ \\
\hline O1 & $0.5384(3)$ & $1.04581(9)$ & $0.8375(2)$ & $0.0462(5)$ \\
\hline N1 & 0.4268 & $0.92521(9)$ & 0.8478 & $0.0343(4)$ \\
\hline $\mathrm{C} 1$ & $0.7655(7)$ & $0.78728(16)$ & $1.0865(7)$ & $0.0924(16)$ \\
\hline $\mathrm{H} 1 \mathrm{~A}$ & 0.6670 & 0.7629 & 1.0890 & $0.111^{*}$ \\
\hline $\mathrm{C} 2$ & $0.9701(7)$ & $0.76567(18)$ & $1.1567(8)$ & $0.117(2)$ \\
\hline $\mathrm{H} 2 \mathrm{~A}$ & 1.0108 & 0.7272 & 1.2078 & $0.140^{*}$ \\
\hline $\mathrm{C} 3$ & $1.1113(6)$ & $0.80149(15)$ & $1.1502(6)$ & $0.0716(11)$ \\
\hline $\mathrm{C} 4$ & $1.0618(5)$ & $0.85845(14)$ & $1.0834(4)$ & $0.0495(7)$ \\
\hline $\mathrm{H} 4 \mathrm{~A}$ & 1.1627 & 0.8827 & 1.0839 & $0.059 *$ \\
\hline C5 & $0.8555(5)$ & $0.87966(12)$ & $1.0140(4)$ & $0.0416(6)$ \\
\hline H5 & 0.8184 & 0.9188 & 0.9670 & $0.050^{*}$ \\
\hline C6 & $0.7048(5)$ & $0.84467(12)$ & $1.0125(4)$ & $0.0411(6)$ \\
\hline $\mathrm{C} 7$ & $0.4795(5)$ & $0.86633(12)$ & $0.9399(4)$ & $0.0429(6)$ \\
\hline $\mathrm{H} 7 \mathrm{~A}$ & 0.3781 & 0.8358 & 0.8598 & $0.051^{*}$ \\
\hline H7B & 0.4629 & 0.8702 & 1.0349 & $0.051^{*}$ \\
\hline $\mathrm{C} 8$ & $0.3497(4)$ & $0.92650(12)$ & $0.6831(4)$ & $0.0382(6)$ \\
\hline $\mathrm{C} 9$ & $0.2963(4)$ & $0.98338(13)$ & 0.5855 & $0.0381(6)$ \\
\hline $\mathrm{C} 10$ & $0.3929(4)$ & $1.03958(13)$ & 0.6678 & $0.0390(6)$ \\
\hline C11 & $0.3352(6)$ & $1.09165(15)$ & 0.5625 & $0.0527(7)$ \\
\hline H11A & 0.3971 & 1.1289 & 0.6147 & $0.063^{*}$ \\
\hline $\mathrm{C} 12$ & $0.1898(6)$ & $1.08905(16)$ & $0.3845(4)$ & $0.0578(8)$ \\
\hline $\mathrm{H} 12 \mathrm{~A}$ & 0.1554 & 1.1243 & 0.3181 & $0.069^{*}$ \\
\hline C13 & $0.0945(5)$ & $1.03431(17)$ & $0.3037(4)$ & $0.0551(8)$ \\
\hline H13A & -0.0052 & 1.0326 & 0.1833 & $0.066^{*}$ \\
\hline $\mathrm{C} 14$ & $0.1479(5)$ & $0.98286(15)$ & 0.4023 & $0.0482(7)$ \\
\hline H14A & 0.0844 & 0.9461 & 0.3468 & $0.058^{*}$ \\
\hline $\mathrm{C} 15$ & $0.3123(6)$ & $0.86788(14)$ & 0.5833 & $0.0566(8)$ \\
\hline H15A & 0.3280 & 0.8756 & 0.4900 & $0.085^{*}$ \\
\hline H15B & 0.1698 & 0.8531 & 0.5359 & $0.085^{*}$ \\
\hline $\mathrm{H} 15 \mathrm{C}$ & 0.4154 & 0.8378 & 0.6608 & $0.085^{*}$ \\
\hline
\end{tabular}
on $\mathrm{F}^{2}$ are statistically about twice as large as those based on F, and R-factors based on ALL data will be even larger.

Fractional atomic coordinates and isotropic or equivalent isotropic displacement parameters $\left(\AA^{2}\right)$ 
Atomic displacement parameters $\left(\AA^{2}\right)$

\begin{tabular}{lllllll}
\hline & $U^{11}$ & $U^{22}$ & $U^{33}$ & $U^{12}$ & $U^{13}$ & $U^{23}$ \\
\hline Pd1 & $0.03017(15)$ & $0.03263(14)$ & $0.03135(14)$ & $-0.00387(10)$ & $0.01650(11)$ & $-0.00210(11)$ \\
F1 & $0.0547(14)$ & $0.0598(14)$ & $0.175(3)$ & $0.0194(11)$ & $0.0329(16)$ & $0.0054(16)$ \\
O1 & $0.0568(12)$ & $0.0479(11)$ & $0.0349(10)$ & $-0.0199(9)$ & $0.0269(9)$ & $-0.0048(8)$ \\
N1 & $0.0332(11)$ & $0.0332(10)$ & $0.0370(11)$ & $-0.0041(8)$ & $0.0203(9)$ & $-0.0044(9)$ \\
C1 & $0.062(2)$ & $0.0424(19)$ & $0.143(4)$ & $-0.0064(17)$ & $0.041(3)$ & $0.027(2)$ \\
C2 & $0.066(3)$ & $0.042(2)$ & $0.183(6)$ & $0.0083(19)$ & $0.036(3)$ & $0.038(3)$ \\
C3 & $0.0486(19)$ & $0.0420(17)$ & $0.089(3)$ & $0.0076(15)$ & $0.0185(19)$ & $-0.0048(17)$ \\
C4 & $0.0444(16)$ & $0.0509(17)$ & $0.0489(17)$ & $0.0005(13)$ & $0.0242(14)$ & $-0.0016(13)$ \\
C5 & $0.0495(16)$ & $0.0367(13)$ & $0.0406(14)$ & $0.0042(12)$ & $0.0269(13)$ & $0.0054(11)$ \\
C6 & $0.0476(15)$ & $0.0316(12)$ & $0.0381(14)$ & $-0.0029(11)$ & $0.0211(12)$ & $-0.0027(11)$ \\
C7 & $0.0504(16)$ & $0.0328(13)$ & $0.0514(16)$ & $-0.0084(11)$ & $0.0327(14)$ & $-0.0041(12)$ \\
C8 & $0.0335(13)$ & $0.0426(14)$ & $0.0395(14)$ & $-0.0059(11)$ & $0.0215(12)$ & $-0.0100(11)$ \\
C9 & $0.0350(13)$ & $0.0478(15)$ & $0.0337(13)$ & $-0.0014(11)$ & $0.0209(11)$ & $-0.0032(11)$ \\
C10 & $0.0424(14)$ & $0.0460(15)$ & $0.0363(14)$ & $-0.0025(12)$ & $0.0271(12)$ & $-0.0016(11)$ \\
C11 & $0.067(2)$ & $0.0483(17)$ & $0.0498(18)$ & $-0.0012(15)$ & $0.0373(17)$ & $0.0029(13)$ \\
C12 & $0.065(2)$ & $0.062(2)$ & $0.0513(18)$ & $0.0141(16)$ & $0.0365(17)$ & $0.0169(16)$ \\
C13 & $0.0447(17)$ & $0.079(2)$ & $0.0360(15)$ & $0.0042(16)$ & $0.0197(13)$ & $0.0037(15)$ \\
C14 & $0.0422(15)$ & $0.0614(18)$ & $0.0375(15)$ & $-0.0046(13)$ & $0.0208(13)$ & $-0.0066(13)$ \\
C15 & $0.070(2)$ & $0.0485(17)$ & $0.0523(18)$ & $-0.0102(15)$ & $0.0360(17)$ & $-0.0160(14)$ \\
& & & & & & \\
\hline
\end{tabular}

Geometric parameters $\left(A,{ }^{\circ}\right)$

\begin{tabular}{llll}
\hline $\mathrm{Pd} 1-\mathrm{O} 1$ & $1.9770(18)$ & $\mathrm{C} 6-\mathrm{C} 7$ & $1.510(4)$ \\
$\mathrm{Pd} 1-\mathrm{O} 1^{\mathrm{i}}$ & $1.9770(18)$ & $\mathrm{C} 7-\mathrm{H} 7 \mathrm{~A}$ & 0.9700 \\
$\mathrm{Pd} 1-\mathrm{N} 1^{\mathrm{i}}$ & $2.028(2)$ & $\mathrm{C} 7-\mathrm{H} 7 \mathrm{~B}$ & 0.9700 \\
$\mathrm{Pd} 1-\mathrm{N} 1$ & $2.028(2)$ & $\mathrm{C} 8-\mathrm{C} 9$ & $1.458(4)$ \\
$\mathrm{F} 1-\mathrm{C} 3$ & $1.369(4)$ & $\mathrm{C} 8-\mathrm{C} 15$ & $1.516(4)$ \\
$\mathrm{O} 1-\mathrm{C} 10$ & $1.321(3)$ & $\mathrm{C} 9-\mathrm{C} 14$ & $1.411(4)$ \\
$\mathrm{N} 1-\mathrm{C} 8$ & $1.297(3)$ & $\mathrm{C} 9-\mathrm{C} 10$ & $1.415(4)$ \\
$\mathrm{N} 1-\mathrm{C} 7$ & $1.474(3)$ & $\mathrm{C} 10-\mathrm{C} 11$ & $1.403(4)$ \\
$\mathrm{C} 1-\mathrm{C} 2$ & $1.378(6)$ & $\mathrm{C} 11-\mathrm{C} 12$ & $1.373(5)$ \\
$\mathrm{C} 1-\mathrm{C} 6$ & $1.381(4)$ & $\mathrm{C} 11-\mathrm{H} 11 \mathrm{~A}$ & 0.9300 \\
$\mathrm{C} 1-\mathrm{H} 1 \mathrm{~A}$ & 0.9300 & $\mathrm{C} 12-\mathrm{C} 13$ & $1.381(5)$ \\
$\mathrm{C} 2-\mathrm{C} 3$ & $1.358(6)$ & $\mathrm{C} 12-\mathrm{H} 12 \mathrm{~A}$ & 0.9300 \\
$\mathrm{C} 2-\mathrm{H} 2 \mathrm{~A}$ & 0.9300 & $\mathrm{C} 13-\mathrm{C} 14$ & $1.363(5)$ \\
$\mathrm{C} 3-\mathrm{C} 4$ & $1.349(5)$ & $\mathrm{C} 13-\mathrm{H} 13 \mathrm{~A}$ & 0.9300 \\
$\mathrm{C} 4-\mathrm{C} 5$ & $1.387(4)$ & $\mathrm{C} 14-\mathrm{H} 14 \mathrm{~A}$ & 0.9300 \\
$\mathrm{C} 4-\mathrm{H} 4 \mathrm{~A}$ & 0.9300 & $\mathrm{C} 15-\mathrm{H} 15 \mathrm{~A}$ & 0.9600 \\
$\mathrm{C} 5-\mathrm{C} 6$ & $1.371(4)$ & $\mathrm{C} 15-\mathrm{H} 15 \mathrm{~B}$ & 0.9600 \\
$\mathrm{C} 5-\mathrm{H} 5$ & 0.9300 & $\mathrm{C} 15-\mathrm{H} 15 \mathrm{C}$ & 0.9600 \\
& & & 108.9 \\
$\mathrm{O} 1-\mathrm{Pd} 1-\mathrm{O} 1^{\mathrm{i}}$ & $180.00(10)$ & $\mathrm{N} 1-\mathrm{C} 7-\mathrm{H} 7 \mathrm{~B}$ & 108.9 \\
$\mathrm{O} 1-\mathrm{Pd} 1-\mathrm{N} 1^{\mathrm{i}}$ & $91.52(8)$ & $\mathrm{C} 6-\mathrm{C} 7-\mathrm{H} 7 \mathrm{~B}$ & 107.7 \\
$\mathrm{O} 1-\mathrm{Pd} 1-\mathrm{N} 1^{\mathrm{i}}$ & $88.48(8)$ & $\mathrm{H} 7 \mathrm{C}-\mathrm{C} 7-\mathrm{H} 7 \mathrm{~B}$ & $122.4(2)$ \\
$\mathrm{O} 1-\mathrm{Pd} 1-\mathrm{N} 1$ & $88.48(8)$ & $\mathrm{N} 1-\mathrm{C} 8-\mathrm{C} 9$ & \\
& & &
\end{tabular}




\begin{tabular}{|c|c|c|c|}
\hline $\mathrm{O} 1-\mathrm{Pd} 1-\mathrm{N} 1$ & $91.52(8)$ & $\mathrm{N} 1-\mathrm{C} 8-\mathrm{C} 15$ & $120.7(3)$ \\
\hline $\mathrm{N} 1{ }^{\mathrm{i}}-\mathrm{Pd} 1-\mathrm{N} 1$ & $180.000(1)$ & $\mathrm{C} 9-\mathrm{C} 8-\mathrm{C} 15$ & $116.9(2)$ \\
\hline $\mathrm{C} 10-\mathrm{O} 1-\mathrm{Pd} 1$ & $118.68(16)$ & $\mathrm{C} 14-\mathrm{C} 9-\mathrm{C} 10$ & $118.1(3)$ \\
\hline $\mathrm{C} 8-\mathrm{N} 1-\mathrm{C} 7$ & $120.0(2)$ & $\mathrm{C} 14-\mathrm{C} 9-\mathrm{C} 8$ & $119.6(3)$ \\
\hline $\mathrm{C} 8-\mathrm{N} 1-\mathrm{Pd} 1$ & $124.82(18)$ & $\mathrm{C} 10-\mathrm{C} 9-\mathrm{C} 8$ & $122.2(2)$ \\
\hline $\mathrm{C} 7-\mathrm{N} 1-\mathrm{Pd} 1$ & $115.13(17)$ & $\mathrm{O} 1-\mathrm{C} 10-\mathrm{C} 11$ & $117.9(3)$ \\
\hline $\mathrm{C} 2-\mathrm{C} 1-\mathrm{C} 6$ & $120.9(4)$ & $\mathrm{O} 1-\mathrm{C} 10-\mathrm{C} 9$ & $124.0(2)$ \\
\hline $\mathrm{C} 2-\mathrm{C} 1-\mathrm{H} 1 \mathrm{~A}$ & 119.6 & $\mathrm{C} 11-\mathrm{C} 10-\mathrm{C} 9$ & $118.1(3)$ \\
\hline $\mathrm{C} 6-\mathrm{C} 1-\mathrm{H} 1 \mathrm{~A}$ & 119.6 & $\mathrm{C} 12-\mathrm{C} 11-\mathrm{C} 10$ & $121.8(3)$ \\
\hline $\mathrm{C} 3-\mathrm{C} 2-\mathrm{C} 1$ & $118.9(4)$ & $\mathrm{C} 12-\mathrm{C} 11-\mathrm{H} 11 \mathrm{~A}$ & 119.1 \\
\hline $\mathrm{C} 3-\mathrm{C} 2-\mathrm{H} 2 \mathrm{~A}$ & 120.6 & $\mathrm{C} 10-\mathrm{C} 11-\mathrm{H} 11 \mathrm{~A}$ & 119.1 \\
\hline $\mathrm{C} 1-\mathrm{C} 2-\mathrm{H} 2 \mathrm{~A}$ & 120.6 & $\mathrm{C} 11-\mathrm{C} 12-\mathrm{C} 13$ & $120.3(3)$ \\
\hline $\mathrm{C} 4-\mathrm{C} 3-\mathrm{C} 2$ & $122.6(3)$ & $\mathrm{C} 11-\mathrm{C} 12-\mathrm{H} 12 \mathrm{~A}$ & 119.9 \\
\hline $\mathrm{C} 4-\mathrm{C} 3-\mathrm{F} 1$ & $118.5(4)$ & $\mathrm{C} 13-\mathrm{C} 12-\mathrm{H} 12 \mathrm{~A}$ & 119.9 \\
\hline $\mathrm{C} 2-\mathrm{C} 3-\mathrm{F} 1$ & $118.9(3)$ & $\mathrm{C} 14-\mathrm{C} 13-\mathrm{C} 12$ & $119.3(3)$ \\
\hline $\mathrm{C} 3-\mathrm{C} 4-\mathrm{C} 5$ & $117.8(3)$ & $\mathrm{C} 14-\mathrm{C} 13-\mathrm{H} 13 \mathrm{~A}$ & 120.3 \\
\hline $\mathrm{C} 3-\mathrm{C} 4-\mathrm{H} 4 \mathrm{~A}$ & 121.1 & $\mathrm{C} 12-\mathrm{C} 13-\mathrm{H} 13 \mathrm{~A}$ & 120.3 \\
\hline $\mathrm{C} 5-\mathrm{C} 4-\mathrm{H} 4 \mathrm{~A}$ & 121.1 & $\mathrm{C} 13-\mathrm{C} 14-\mathrm{C} 9$ & $122.4(3)$ \\
\hline $\mathrm{C} 6-\mathrm{C} 5-\mathrm{C} 4$ & $121.9(3)$ & $\mathrm{C} 13-\mathrm{C} 14-\mathrm{H} 14 \mathrm{~A}$ & 118.8 \\
\hline $\mathrm{C} 6-\mathrm{C} 5-\mathrm{H} 5$ & 119.0 & $\mathrm{C} 9-\mathrm{C} 14-\mathrm{H} 14 \mathrm{~A}$ & 118.8 \\
\hline $\mathrm{C} 4-\mathrm{C} 5-\mathrm{H} 5$ & 119.0 & $\mathrm{C} 8-\mathrm{C} 15-\mathrm{H} 15 \mathrm{~A}$ & 109.5 \\
\hline $\mathrm{C} 5-\mathrm{C} 6-\mathrm{C} 1$ & $117.9(3)$ & $\mathrm{C} 8-\mathrm{C} 15-\mathrm{H} 15 \mathrm{~B}$ & 109.5 \\
\hline $\mathrm{C} 5-\mathrm{C} 6-\mathrm{C} 7$ & $123.5(2)$ & $\mathrm{H} 15 \mathrm{~A}-\mathrm{C} 15-\mathrm{H} 15 \mathrm{~B}$ & 109.5 \\
\hline $\mathrm{C} 1-\mathrm{C} 6-\mathrm{C} 7$ & $118.6(3)$ & $\mathrm{C} 8-\mathrm{C} 15-\mathrm{H} 15 \mathrm{C}$ & 109.5 \\
\hline $\mathrm{N} 1-\mathrm{C} 7-\mathrm{C} 6$ & $113.4(2)$ & $\mathrm{H} 15 \mathrm{~A}-\mathrm{C} 15-\mathrm{H} 15 \mathrm{C}$ & 109.5 \\
\hline $\mathrm{N} 1-\mathrm{C} 7-\mathrm{H} 7 \mathrm{~A}$ & 108.9 & $\mathrm{H} 15 \mathrm{~B}-\mathrm{C} 15-\mathrm{H} 15 \mathrm{C}$ & 109.5 \\
\hline $\mathrm{C} 6-\mathrm{C} 7-\mathrm{H} 7 \mathrm{~A}$ & 108.9 & & \\
\hline $\mathrm{N} 1{ }^{\mathrm{i}}-\mathrm{Pd} 1-\mathrm{O} 1-\mathrm{C} 10$ & $-134.7(2)$ & $\mathrm{Pd} 1-\mathrm{N} 1-\mathrm{C} 8-\mathrm{C} 9$ & $-3.2(4)$ \\
\hline $\mathrm{N} 1-\mathrm{Pd} 1-\mathrm{O} 1-\mathrm{C} 10$ & $45.3(2)$ & $\mathrm{C} 7-\mathrm{N} 1-\mathrm{C} 8-\mathrm{C} 15$ & $0.2(4)$ \\
\hline $\mathrm{O} 1-\mathrm{Pd} 1-\mathrm{N} 1-\mathrm{C} 8$ & $-25.1(2)$ & $\mathrm{Pd} 1-\mathrm{N} 1-\mathrm{C} 8-\mathrm{C} 15$ & $176.5(2)$ \\
\hline $\mathrm{O} 1{ }^{\mathrm{i}}-\mathrm{Pd} 1-\mathrm{N} 1-\mathrm{C} 8$ & $154.9(2)$ & $\mathrm{N} 1-\mathrm{C} 8-\mathrm{C} 9-\mathrm{C} 14$ & $-157.8(3)$ \\
\hline $\mathrm{O} 1-\mathrm{Pd} 1-\mathrm{N} 1-\mathrm{C} 7$ & $151.39(18)$ & $\mathrm{C} 15-\mathrm{C} 8-\mathrm{C} 9-\mathrm{C} 14$ & $22.6(4)$ \\
\hline $\mathrm{O} 1{ }^{\mathrm{i}}-\mathrm{Pd} 1-\mathrm{N} 1-\mathrm{C} 7$ & $-28.61(18)$ & $\mathrm{N} 1-\mathrm{C} 8-\mathrm{C} 9-\mathrm{C} 10$ & $24.0(4)$ \\
\hline $\mathrm{C} 6-\mathrm{C} 1-\mathrm{C} 2-\mathrm{C} 3$ & $-0.8(9)$ & $\mathrm{C} 15-\mathrm{C} 8-\mathrm{C} 9-\mathrm{C} 10$ & $-155.6(3)$ \\
\hline $\mathrm{C} 1-\mathrm{C} 2-\mathrm{C} 3-\mathrm{C} 4$ & $2.6(9)$ & $\mathrm{Pd} 1-\mathrm{O} 1-\mathrm{C} 10-\mathrm{C} 11$ & $141.1(2)$ \\
\hline $\mathrm{C} 1-\mathrm{C} 2-\mathrm{C} 3-\mathrm{F} 1$ & $-179.5(5)$ & $\mathrm{Pd} 1-\mathrm{O} 1-\mathrm{C} 10-\mathrm{C} 9$ & $-40.7(3)$ \\
\hline $\mathrm{C} 2-\mathrm{C} 3-\mathrm{C} 4-\mathrm{C} 5$ & $-2.3(7)$ & $\mathrm{C} 14-\mathrm{C} 9-\mathrm{C} 10-\mathrm{O} 1$ & $-178.0(3)$ \\
\hline $\mathrm{F} 1-\mathrm{C} 3-\mathrm{C} 4-\mathrm{C} 5$ & $179.8(3)$ & $\mathrm{C} 8-\mathrm{C} 9-\mathrm{C} 10-\mathrm{O} 1$ & $0.2(4)$ \\
\hline $\mathrm{C} 3-\mathrm{C} 4-\mathrm{C} 5-\mathrm{C} 6$ & $0.2(5)$ & $\mathrm{C} 14-\mathrm{C} 9-\mathrm{C} 10-\mathrm{C} 11$ & $0.2(4)$ \\
\hline $\mathrm{C} 4-\mathrm{C} 5-\mathrm{C} 6-\mathrm{C} 1$ & $1.4(5)$ & $\mathrm{C} 8-\mathrm{C} 9-\mathrm{C} 10-\mathrm{C} 11$ & $178.4(3)$ \\
\hline $\mathrm{C} 4-\mathrm{C} 5-\mathrm{C} 6-\mathrm{C} 7$ & $179.4(3)$ & $\mathrm{O} 1-\mathrm{C} 10-\mathrm{C} 11-\mathrm{C} 12$ & $178.2(3)$ \\
\hline $\mathrm{C} 2-\mathrm{C} 1-\mathrm{C} 6-\mathrm{C} 5$ & $-1.1(7)$ & $\mathrm{C} 9-\mathrm{C} 10-\mathrm{C} 11-\mathrm{C} 12$ & $-0.2(5)$ \\
\hline $\mathrm{C} 2-\mathrm{C} 1-\mathrm{C} 6-\mathrm{C} 7$ & $-179.1(5)$ & $\mathrm{C} 10-\mathrm{C} 11-\mathrm{C} 12-\mathrm{C} 13$ & $0.4(5)$ \\
\hline $\mathrm{C} 8-\mathrm{N} 1-\mathrm{C} 7-\mathrm{C} 6$ & $87.6(3)$ & $\mathrm{C} 11-\mathrm{C} 12-\mathrm{C} 13-\mathrm{C} 14$ & $-0.7(5)$ \\
\hline $\mathrm{Pd} 1-\mathrm{N} 1-\mathrm{C} 7-\mathrm{C} 6$ & $-89.0(2)$ & $\mathrm{C} 12-\mathrm{C} 13-\mathrm{C} 14-\mathrm{C} 9$ & $0.8(5)$ \\
\hline $\mathrm{C} 5-\mathrm{C} 6-\mathrm{C} 7-\mathrm{N} 1$ & $8.3(4)$ & $\mathrm{C} 10-\mathrm{C} 9-\mathrm{C} 14-\mathrm{C} 13$ & $-0.6(4)$ \\
\hline
\end{tabular}


$\mathrm{C} 1-\mathrm{C} 6-\mathrm{C} 7-\mathrm{N} 1 \quad-173.7$ (3)

$\mathrm{C} 7-\mathrm{N} 1-\mathrm{C} 8-\mathrm{C} 9$
$\mathrm{C} 8-\mathrm{C} 9-\mathrm{C} 14-\mathrm{C} 13$

$-178.8(3)$

Symmetry code: (i) $-x+1,-y+2,-z+2$.

Hydrogen-bond geometry $\left(A,{ }^{\circ}\right)$

\begin{tabular}{lllll}
\hline$D-\mathrm{H} \cdots A$ & $D-\mathrm{H}$ & $\mathrm{H} \cdots A$ & $D \cdots A$ & $D-\mathrm{H} \cdots A$ \\
\hline $\mathrm{C} 4-\mathrm{H} 4 A \cdots \mathrm{O} 1^{\mathrm{ii}}$ & 0.93 & 2.50 & $3.405(5)$ & 165 \\
\hline
\end{tabular}

Symmetry code: (ii) $-x+2,-y+2,-z+2$. 